\title{
FORMACIÓN UNIVERSITARIA EN UN MUNDO GLOBALIZADO Y CAMBIANTE: \\ El Programa Académico de Bachillerato de la Universidad de Chile
}

\author{
Julio Mella* \\ Luis Menke* \\ Jorge Babul*
}




\section{RESUMEN}

Los permanentes cambios de la sociedad actual obligan al sistema educacional a propiciar nuevos espacios para dar respuesta a las demandas que se van presentando. Con esta finalidad, la Universidad de Chile creó el Programa Académico de Bachillerato. Para su inserción en el sistema universitario fue necesario articular planes de estudio y proponer discusiones en torno a cómo se integrarían los estudios de pregrado, lo que ha generado las pautas para nuevas propuestas al interior de esta casa de estudios. Como resultado, se ha logrado crear un espacio académico donde confluyen diversas áreas del saber, que apoya a los estudiantes en su exploración vocacional y que genera sujetos mejor preparados para enfrentar los estudios superiores. El Programa de Bachillerato ha hecho aportes de gran valor y ha generado también los nuevos desafíos que se deben enfrentar.

\section{ABSTRACT}

The permanent changes in society at present force the educational system to favor the creation of new spaces to answer to rising demands. To this end, the Universidad de Chile created the Baccalaureate Academic Program. In order to make it part of the university system it was necessary to prepare curricula, and encourage debates on how the program would be integrated to undergraduate studies; this has given rise to the guidelines for new proposals at the University. Consequently, an academic space has been created where several areas of knowledge come together, and this provides support to students in their vocational search, thus creating individuals who are better prepared to tackle higher education. The Baccalaureate Program has made highly valuable contributions and has also generated new challenges that need to be addressed. 


\title{
FORMACIÓN UNIVERSITARIA EN UN MUNDO GLOBALIZADO Y CAMBIANTE:
}

\author{
El Programa Académico de Bachillerato de la Universidad \\ de Chile
}

\section{EDUCACIÓN SUPERIOR Y CALIDAD}

El concepto de cambio se ha ido incorporando cada vez con mayor naturalidad a la vida cotidiana. La sociedad de hoy está constantemente modificándose, tanto por la introducción de nuevas tecnologías, la globalización, las variaciones económicas y políticas, como por la búsqueda de soluciones a los problemas que nacen de estos acontecimientos.

Este cambio es vertiginoso y generalmente impredecible; sus repercusiones se extienden a todos los ámbitos del saber y del hacer del hombre. Nacen constantemente nuevas formas y perspectivas de vida, nuevas necesidades y nuevas soluciones; nacen nuevos sujetos, con inquietudes e intereses en permanente transformación.

En este escenario es imprescindible que constantemente se replantee la educación, en todos sus niveles, para que no quede descontextualizada de su entorno. Se debe revisar si efectivamente se hace lo que se cree que se está haciendo, si se está respondiendo a lo que se quiere responder y a las acciones que se necesitan en un momento en particular.

En las últimas décadas el mundo de la educación ha registrado un giro gradual y pronunciado en su orientación. La introducción de reformas en la enseñanza básica y media, los cambios en el espectro de oferta en la educación superior, potenciados por la proliferación de universidades privadas, y la existencia de estudiantes con nuevos marcos conceptuales de referencia, han cambiado el mercado de la educación y han cuestionado lo que se estaba ofreciendo hasta ahora. El énfasis que antes estuviera puesto en la formación de profesionales, según una oferta de carreras tradicionales para un mercado tradicional, se ha ido modificando. Las universidades han incrementado sus 
esfuerzos en considerar las necesidades y deseos de los estudiantes, especialmente teniendo presente las condiciones en las que llegan de la educación media. Como consecuencia de esto, muchas casas de estudio han desarrollado opciones para el encuentro de esas necesidades, surgiendo así los programas de Bachillerato. Frente a ese modelo y al considerar ahora los cambios que se han dado en distintos niveles de lo social, cabe plantearse cómo se han desarrollado los programas de Bachillerato en el medio educativo y cuáles serán las perspectivas que seguirán orientando su quehacer.

\section{LOS INICIOS DEL BACHILLERATO}

Un mundo que permanentemente revisa y cuestiona paradigmas exige individuos flexibles que, más allá de albergar y perpetuar conocimientos específicos, sean capaces de modificar su posición con la misma facilidad con que puedan lograr una nueva incorporación de saberes y técnicas. Un mundo de comunicaciones veloces y globalizadas plantea nuevas exigencias a la educación, que apunten a una formación básica capaz de entregar elementos de análisis y criterios, que no se limiten a una disciplina o a un problema en particular.

Por otra parte, no sólo se hace necesario preparar al estudiante para la realidad en la que se insertará profesionalmente, sino también para ayudarlo a situar su posición actual en el mundo que lo rodea. Más aún porque los problemas sociales de hoy no se entienden unidimensionalmente. Para poder abarcar los fenómenos y comprender sus repercusiones, es menester incluir la mayor cantidad posible de elementos de análisis, en un todo coherente e integrado. Para dar soluciones viables a un país en desarrollo, las disciplinas del conocimiento deben actuar en conjunto y un sujeto que hace aportes a esa realidad, debe ser capaz de entender y utilizar dicha riqueza. Es necesario, entonces, el tránsito hacia una educación globalizada, donde exista el espacio para el saber actual y dinámico, pero donde a la vez quepan diversas áreas de conocimientos, que se encuentren, interactúen y dialoguen.

Sin embargo, una consecuencia de la inestabilidad y multiplicidad de los saberes es el problema que surge cuando un estudiante se ve 
enfrentado por primera vez a elegir entre ellos. En el sistema educacional y formativo actual se produce la paradoja de que tras años de una rígida estructura escolar, el sujeto se enfrenta a tomar una decisión que aparentemente determinará el resto de su vida, y las opciones que se le presentan parecen ser excluyentes unas de otras. Al egresado de la enseñanza media se le obliga a decidir sobre estereotipos de vida, con un rol y un estatus en la sociedad, ligados a una carrera en particular, y se le obliga a apostar a que ellos se mantendrán hasta que salga al mundo profesional. La consecuencia es un alto nivel de presión sobre los estudiantes, donde el "fracaso" por una mala elección se les traspasa como problema individual de orientación vocacional y se traduce, a la larga, en la aparición de sujetos inseguros y con baja autoestima.

El problema de la deserción no es un tema menor en la Universidad, considerando que, en el año 2001, de los 136.000 estudiantes que ingresaron al sistema universitario 30.000 abandonaron antes de que culminara el segundo semestre, lo que representa una deserción del $22 \%$. Por su parte, el sistema se encarga de mantener el círculo "castigando" el cambio de intereses vocacionales, con consecuencias tales como tener que recomenzar por las pruebas de ingreso a la Universidad u obligando a los alumnos a cargar de por vida con una profesión que no se quiere o se quiere menos que otra.

Atento a todos estos aspectos, con un espíritu de integración de disciplinas más que de segregación, buscando dar una repuesta innovadora a las crecientes necesidades no cubiertas y enfatizando el aspecto formativo por sobre el profesionalizante, se estructura el Programa Académico de Bachillerato en la Universidad de Chile.

Desde su origen se confieren a este programa características que buscan satisfacer el propósito de la transdisciplinariedad, tales como la arriesgada propuesta de un ingreso indiferenciado, tanto para quienes tengan inclinaciones científicas como para aquellos que las tengan humanistas. Es riesgoso, asumiendo que el fraccionamiento del conocimiento en áreas es artificial, pero que la cultura en general y la cultura educacional en particular parcelan y etiquetan a los estudiantes desde temprano. 
Se diseñó un programa de estudios que abarcara las materias con mayor presencia en los planes de las carreras de la Universidad de Chile, que permitiera al estudiante comprender las ideas centrales y modos de pensar propios de dichas disciplinas. Así, se pretendió garantizar la formación general del universitario y, a su vez, velar por la homologación de las materias en la continuación de estudios al interior de la Universidad.

Por otro lado, la implementación del plan de estudios del Bachillerato requirió consensuar no sólo las disciplinas sino también a los docentes que las impartían. Este ordenamiento se debió hacer primero al interior de cada materia, para que luego se pudieran encontrar y entonces formar un proyecto educativo coherente y flexible, capaz de adaptarse a las particularidades e intereses de cada estudiante.

Un elemento que exigió una discusión prolongada fue la falta de articulación estructural de los planes de estudio de las diversas facultades al interior de la Universidad. Esto a propósito de cómo se daría la transición desde el Bachillerato a una carrera de destino. Para este efecto se tuvo que revisar cómo se harían las homologaciones y se creó, además, un sistema de vacantes internas en cada una de las carreras para los graduados del programa. Con el tiempo también fue necesario diseñar y perfeccionar mecanismos que facilitaran la selección de los estudiantes que harían uso de esas vacantes.

Una vez resueltos aspectos de contenido y de forma se presenta el Bachillerato como una alternativa innovadora de ingreso a la educación superior.

\section{A CASI DIEZ AÑOS DE LA PUESTA EN MARCHA DEL PROGRAMA}

Al Programa Académico de Bachillerato en la Universidad de Chile se le asignaron inicialmente tres objetivos:

a) Permitir al estudiante profundizar en disciplinas que sirven de fundamento a los estudios académicos o profesionales, evitando así la deserción que se produce en las carreras como consecuencia de una elección equivocada o una preparación inapropiada;

b) Atenuar la desigualdad de oportunidades de acceso a la Universidad;

c) Evitar una profesionalización prematura, necesariamente limitada. 
La recepción del programa dentro de la comunidad universitaria ha pasado por varios momentos. El principio estuvo marcado por la expectativa de si esta alternativa era o no viable. Como muchos cambios en estructuras que han permanecido invariables por muchos años, se le llegó a mirar con cierta desconfianza e incluso con temor en algunos sectores. Era cuestionable si la situación de la Universidad, en aquel entonces, permitiría la inserción de un sistema alternativo de incorporación a las carreras y si el programa no se convertiría en una opción de segunda clase, para estudiantes no calificados ni dignos de carreras de prestigio. No obstante, con el tiempo, el Programa de Bachillerato se fue consolidando y junto con las experiencias en otras universidades, fue mostrando resultados positivos.

Es un hecho que la postulación al Programa de Bachillerato es altamente considerada por los estudiantes que egresan de la educación media y el que se haya instalado en el imaginario de posibilidades, ha marcado sin duda el primer paso en la aceptación y consolidación del proyecto. En la actualidad el Bachillerato es la carrera más señalada entre las postulaciones de los estudiantes que rinden la Prueba de Aptitud Académica (PAA), lo que indica que un alto porcentaje está dispuesto a ingresar a él.

Por otra parte, al analizar la deserción de los primeros años de las carreras en general, es posible comprobar que la indecisión vocacional y la formación deficitaria con la que llegan los estudiantes, han sido elementos de los que había que hacerse cargo de una forma más activa y no sólo a través de propuestas individuales y aisladas. Desde la Universidad, como institución, el Programa de Bachillerato se presenta como una respuesta concreta para quienes no habían tenido un espacio real que los acogiera.

Por otro lado, al exponer a los estudiantes a un alto nivel académico y ofrecer simultáneamente la posibilidad de ir explorando diversas áreas del saber, se ha ido logrando perfilar un tipo particular de estudiante al interior de la Universidad -y así se ha percibido por varios sectores de la comunidad académica-, no sólo con una formación más global frente a los fenómenos, sino también con un nivel de estudio que le permite enfrentarse de mejor manera a sus materias. Los estudios de seguimiento realizados demuestran que el 
rendimiento de los estudiantes del programa, en las carreras a las que se incorporan, es igual o superior al de quienes ingresaron vía PAA, incluso en carreras de alta demanda. Ello cobra mayor relevancia si se considera que algunos no habrían ingresado directamente a la carrera debido a bajo puntaje en la PAA. Respecto de este punto también es posible constatar que ha habido un cambio en el alumno que se incorpora al programa. Si una de las preocupaciones originales era el hecho de que los estudiantes menos calificados iban a entrar a una carrera, es precisamente el aumento en los puntajes de ingreso al programa el dato que puede ayudar a desmitificar dicha premisa. Desde 1995, segundo año de implementación del Programa Académico de Bachillerato en la Universidad de Chile, hasta ahora, el puntaje del último ingresado se incrementó en 63 puntos, en tanto que el puntaje máximo de ingreso para el año 2002 fue de 774 puntos ( véase cuadro 1).

\section{Cuadro 1}

Puntajes de ingreso de los estudiantes matriculados en el programa

\begin{tabular}{|ccc|}
\cline { 2 - 3 } \multicolumn{1}{c|}{} & \multicolumn{2}{c|}{ Puntaje de ingreso } \\
\hline Año de ingreso & Máximo & Mínimo $^{1}$ \\
1994 & 751.80 & $650.20^{2}$ \\
1995 & 730.20 & 620.20 \\
1996 & 739.50 & 639.40 \\
1997 & 734.10 & 660.80 \\
1998 & 749.90 & 663.50 \\
1999 & 757.80 & 668.80 \\
2000 & 750.30 & 675.00 \\
2001 & 753.70 & 678.30 \\
2002 & 774.60 & 683.60 \\
\hline
\end{tabular}

Por otro lado, cuando se considera que los estudiantes recién egresados de la enseñanza media tienden a desertar de su carrera por indecisión o cambio vocacional, es alentador comprobar que el Programa de Bachillerato ha aumentado año tras año el porcentaje de

1. Según la ponderación: 30\% PA Verbal, 30\% PA Matemática, 10\% P. Historia y Geografía y $30 \%$ NEM.

2. En 1994 el requisito mínimo de postulación fue 650 puntos. 
estudiantes que ingresan por primera vez a la Universidad: de un 54\% en 1994 a un 70\% en el 2002. Esto significa que los alumnos, ante la perspectiva de "fracasar" en una carrera, optan por consolidar conocimientos y clarificar su vocación en el programa. El cuadro 2 muestra cómo ha ido variando el número de estudiantes que egresan de la enseñanza media para ingresar inmediatamente al programa.

\section{Cuadro 2}

Año de egreso de la enseñanza media de los estudiantes matriculados en el programa

\begin{tabular}{|ccc|}
\cline { 2 - 3 } \multicolumn{1}{c|}{} & \multicolumn{2}{c|}{ Egreso de la educación media } \\
\hline Año de ingreso & Del año & Años anteriores \\
1994 & $54 \%$ & $46 \%$ \\
1995 & $62 \%$ & $38 \%$ \\
1996 & $63 \%$ & $37 \%$ \\
1997 & $70 \%$ & $30 \%$ \\
1998 & $76 \%$ & $24 \%$ \\
1999 & $77 \%$ & $23 \%$ \\
2000 & $76 \%$ & $24 \%$ \\
2001 & $70 \%$ & $30 \%$ \\
2002 & $70 \%$ & $30 \%$ \\
\hline
\end{tabular}

El Programa de Bachillerato también ha ayudado a los estudiantes a conocer nuevas opciones y a sugerirles la posibilidad de ingresar a carreras diferentes de las que tradicionalmente se conocen al momento de egresar de la educación media. Esta ignorancia no sólo está influida por falta de información sobre otras carreras; también deriva de fallas en las metodologías de enseñanza, que vinculan profesiones con disciplinas aburridas e incomprensibles. Alrededor del $80 \%$ de los estudiantes que ingresa al programa reporta preferencia por no más de 10 carreras. Sin embargo, el año 2002, los graduados de Bachiller ingresaron a 31 carreras diferentes dentro de la Universidad.

La transición entre la educación media y la superior no es todo lo fluida que debería ser; sin embargo, a la luz de la experiencia se puede comprobar que el Programa de Bachillerato cumple un rol en dicho proceso, facilitando el tránsito. 


\section{EL FUTURO DE LA EDUCACIÓN}

Si las ciencias exactas y naturales se complementaran exitosamente con las ciencias sociales y las humanidades, las profesiones y la educación superior en general se revitalizarían. Las instituciones y los individuos necesitan de instancias para observarse y reflexionar sobre su futuro, a fin de trazar nuevas estrategias e incorporar reformas importantes que aporten al país.

El ingreso del Bachillerato al espectro educativo ha favorecido la reflexión en la Universidad de Chile y ha permitido pensar en reformas a los estudios de pregrado, apuntando a globalizar los programas de estudio de todas las carreras. Esta innovación curricular permitirá que en toda la Universidad se replique parte del modelo, en el que diversas disciplinas tengan espacios comunes desde donde un estudiante las pueda integrar.

Sin embargo, esta reflexión debe ser constante y debe anticiparse a los distintos problemas que enfrentará la educación en los años venideros. En ese escenario, el Bachillerato tiene un rol preponderante en la investigación y propuesta de modelos educativos de trascendencia para la Universidad en su totalidad. Es necesario que exista una instancia capaz de estar atenta a la situación de los estudios de pregrado; que diseñe modos alternativos de inserción de propuestas educativas; que defina nuevos requerimientos para los docentes universitarios, en el contexto de una carrera académica; que estimule el permanente perfeccionamiento y calidad de la docencia; que reexamine los sistemas de selección de alumnos y las demandas que debe satisfacer el sistema de educación media; que defina los nuevos requerimientos para las estructuras institucionales universitarias, para que funcionen como instancias facilitadoras del diálogo permanente en materias del pregrado y que, en fin, se constituya como un espacio de encuentro para el pensar la educación y tomar las decisiones adecuadas.

Los desafíos aún son múltiples y la educación superior está implementando nuevos proyectos. Los programas de Bachillerato pueden ser una instancia de experimentación y un espacio de reflexión para la educación superior. Si estos espacios no se propician, el riesgo 
es encontrarse con una educación obsoleta, en cuyo contexto otros medios se apropien de la tarea que le corresponde a la Universidad y que ésta no asuma su responsabilidad para con los estudiantes y las necesidades del país. 